\section{5-bit, 10 GSamples/s track-and-hold circuit with input feedthrough cancellation}

\section{I.-H. Wang, J.-L. Lin and S.-I. Liu}

Input signal feedthrough in a high-speed track-and-hold $(\mathrm{T} / \mathrm{H})$ circuit often degrades the performance of analogue-to-digital data converters. A 10 GSamples/s CMOS $\mathrm{T} / \mathrm{H}$ circuit with input feedthrough cancellation is proposed. This $\mathrm{T} / \mathrm{H}$ circuit has been fabricated in $0.18 \mu \mathrm{m}$ CMOS process. It achieves 5-bit resolution in $2.5 \mathrm{GHz}$ analogue input signal at 10 GSamples/s.

Introduction: A high-speed track-and-hold $(\mathrm{T} / \mathrm{H})$ circuit plays an important role in high-speed analogue-to-digital converters (ADCs) [1]. In conventional high-speed $\mathrm{T} / \mathrm{H}$ circuits, the parasitic capacitances in the active devices introduce the parasitic signal paths, which degrade the circuit performances. In [2], the complementary clocks and the parasitic capacitance cancellation circuit are used to alleviate the clock feedthrough, the charge injection, and the parasitic capacitance to enhance the linearity and bandwidth. However, the input signal feedthrough [3] still affects the output signal in the hold mode. In this Letter, a new $\mathrm{T} / \mathrm{H}$ circuit is proposed with input feedthrough cancellation.

Circuit description: The proposed $\mathrm{T} / \mathrm{H}$ circuit is shown in Fig. 1. This $\mathrm{T} / \mathrm{H}$ circuit uses the dual differential input sampling circuit and a wideband analogue adder to cancel the input signal feedthrough in the hold mode. In an open-loop $\mathrm{T} / \mathrm{H}$ circuit, the trade-off between the turn-on resistance of the switch and the hold capacitor limits the speed and accuracy. The $\mathrm{T} / \mathrm{H}$ acquisition time constant in the tracking mode is given as

$$
\tau=R_{\mathrm{on}} C_{H}=\frac{C_{H}}{\mu_{n} C_{o x}(W / L)\left(V_{G S}-V_{T H}\right)}
$$

where $R_{\mathrm{on}}$ and $C_{H}$ are the turn-on resistance of the switch and the hold capacitor, respectively. According to (1), it achieves a higher bandwidth to reduce the capacitor and the resistance. In the hold mode, the pedestal error due to the charge injection is given as

$$
\Delta V_{p}=\frac{Q_{\text {channel }}}{2 C_{H}}=\frac{W L C_{o x}\left(V_{G S}-V_{T H}\right)}{2 C_{H}}
$$

where $Q_{\text {channel }}$ are the charge stored in the MOS transistor channel. Equation (2) shows that it reduces the pedestal error to increase the hold capacitor and switch size. There is a trade-off to choose the hold capacitance and switch size. Based on (1) and (2), the hold capacitor of $120 \mathrm{fF}$ and the aspect ratio $W / L=22.5 \mu \mathrm{m} / 0.18 \mu \mathrm{m}$ of the MOSFET are chosen.

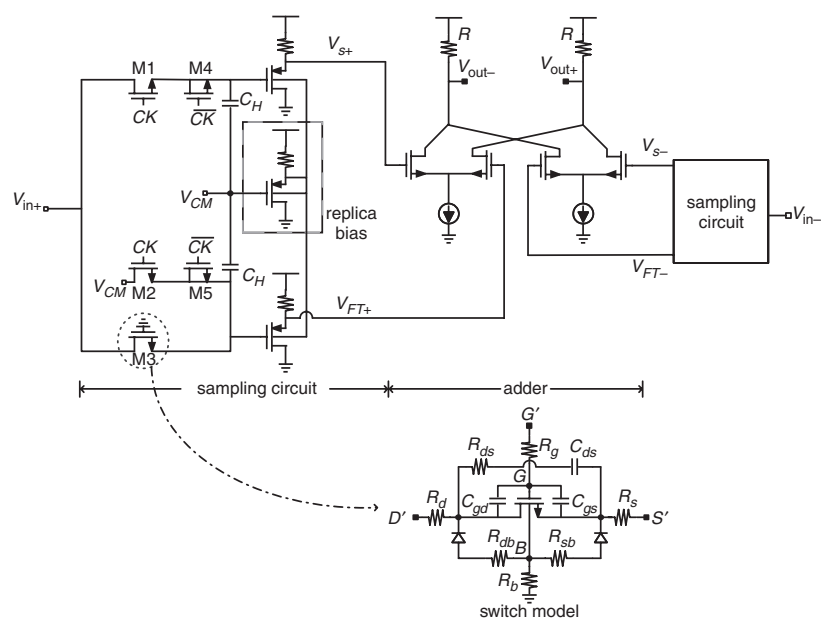

Fig. 1 Proposed track-and-hold circuit

Moreover, the input feedthrough introduces the undesired signal on the small hold capacitor and it degrades the resolution of the $\mathrm{T} / \mathrm{H}$ circuit. The high frequency MOS model with parasitic components is also given in Fig. 1. There are two major input feedthrough paths. The first one is the well resistance, $R_{d s}$, series with the junction capacitance, $C_{d s}$. The other is through the overlap capacitances, $C_{g s}$ and $C_{g d}$. The impedance between the drain and source of this MOS model is given as

$$
Z(s)=\frac{s R_{d s} C_{d s}\left(C_{g s}+C_{g d}\right)+\left(C_{g s}+C_{g d}\right)}{s^{2} R_{d s} C_{d s} C_{g s} C_{g d}+s\left[C_{d s}\left(C_{g s}+C_{g d}\right)+C_{g d} C_{g s}\right]}
$$

According to (3), the higher analogue input frequency and shorter MOS channel length make $Z(s)$ smaller. In the hold mode, the feedthrough current is increased owing to this lower impedance. To deal with this effect, the proposed $\mathrm{T} / \mathrm{H}$ circuit adopts a master switch, $\mathrm{M} 1$, to sample the input signal in the tracking mode. And the switch, M2, is used to reset the input feedthrough voltage to the common voltage, $V_{C M}$, in the tracking mode [3]. The replica switch, M3, which is always off, is used to sample the input feedthrough in the hold mode. Two transistors, M4 and M5, are used to compensate the clock feedthrough charges.

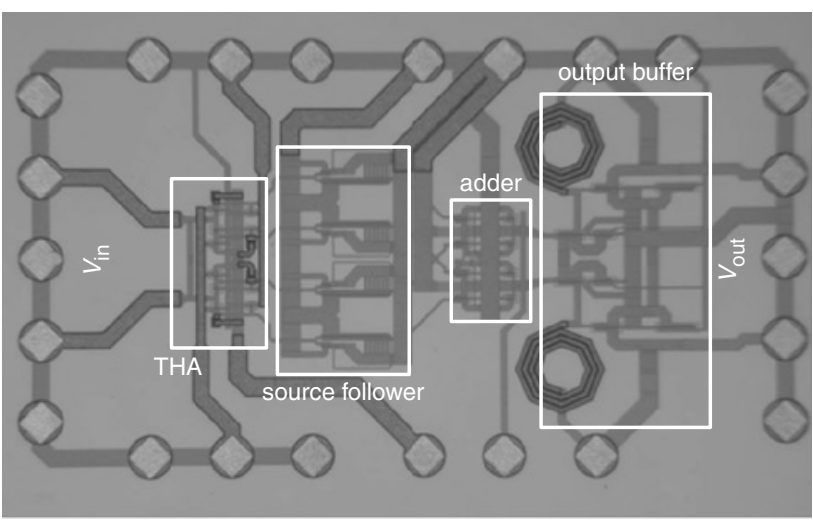

Fig. 2 Die photo
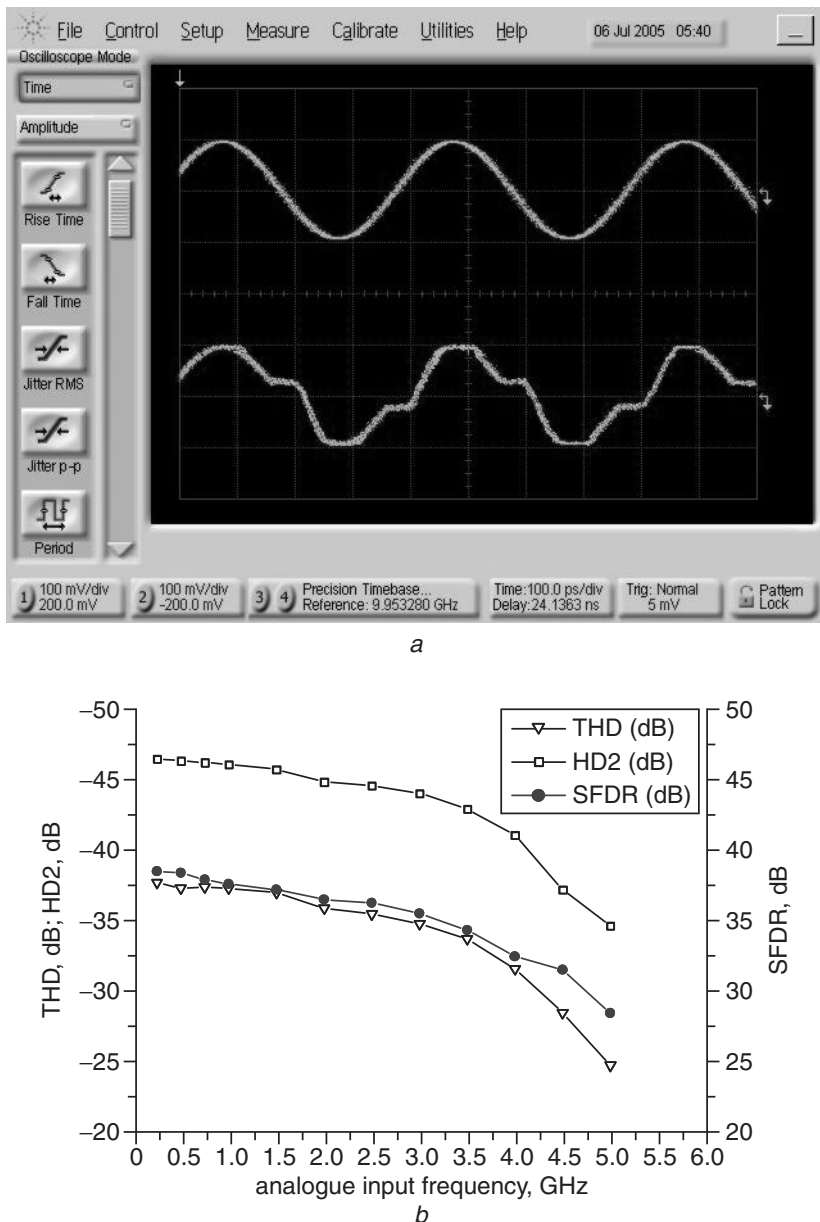

Fig. 3 Measured transient response for $2.5 \mathrm{GHz}$ input signal at 10 GSamples/s

$a$ Measured transient response

$b$ Measured THD and SFDR 
The source follower with replica-based well-biasing [4] is used to drive the following analogue adder. The wideband analogue adder is implemented by two source-coupled pairs to achieve the wide bandwidth and high linearity. The adder is used to cancel the undesired input feedthrough voltage. Based on simulation results, the output signal-tonoise distortion ratio is improved from 22.5 to $49 \mathrm{~dB}$ by the proposed input feedthrough cancellation.

In order to drive the instrument, the wideband output buffer is realised by two cascaded source-coupled pairs: the first source-coupled pair with the conventional inductor shunt-peaking technique to enhance signal bandwidth; the second source-coupled pair with only resistance loads to match the output impedance.

Experimental results: The proposed $\mathrm{T} / \mathrm{H}$ circuit has been fabricated in $0.18 \mu \mathrm{m}$ CMOS technology and measured in die-on-board assemblies. Fig. 2 shows the die photo. The total chip area is $1.5 \times 0.82 \mathrm{~mm}^{2}$. The transient responses are measured by using Agilent N4901A to generate the sampling clock and synchronise with the signal generator E4422B and the sampling oscilloscope $86100 \mathrm{C}$. The frequency of the analogue sinusoidal input is varied from $250 \mathrm{MHz}$ to $2.5 \mathrm{GHz}$. A typical transient response is given in Fig. $3 a$ with the analogue input frequency of $2.5 \mathrm{GHz}$ at 10GSamples/s. The measured spurious-free dynamic range (SFDR), the total harmonic distortion (THD) and the second-harmonic distortion (HD2) are shown in Fig. $3 b$. The measurement results reveal that the $\mathrm{T} / \mathrm{H}$ circuit achieves 5-bit effective number of bits $(\mathrm{ENOB})$ at $10 \mathrm{GSamples} / \mathrm{s}$. Moreover, the beat frequency $(\Delta f)$ of $1 \mathrm{MHz}$ is also measured using $5.001 \mathrm{GHz}$ analogue input signal with the sampling clock of $5 \mathrm{GHz}$, the THD is smaller than $-34 \mathrm{~dB}$. Table 1 summarises the measured performance and comparisons with previous works.

Table 1: Performance summary

\begin{tabular}{|c|c|c|c|c|}
\hline & {$[2]$} & {$[5]$} & {$[6]$} & This work \\
\hline Technology & $90 \mathrm{~nm}$ CMOS & SiGe HBT & InP DHBT & $0.18 \mu \mathrm{m}$ CMOS \\
\hline Sampling rate & 4 GSamples/s & 8 GSamples/s & 12 GSamples/s & 10 GSamples/s \\
\hline $\begin{array}{c}\text { Analogue } \\
\text { input } \\
\text { frequency }\end{array}$ & $4 \mathrm{GHz}$ & $4 \mathrm{GHz}$ & $2 \mathrm{GHz}$ & $250 \mathrm{MHz}-5 \mathrm{GHz}$ \\
\hline THD (dB) & -27 & -30 & -31 & -37.7 to -24.7$)$ \\
\hline SFDR (dB) & - & 30 & - & $38.4-28.4$ \\
\hline $\begin{array}{c}\text { Supply } \\
\text { voltage }(\mathrm{V})\end{array}$ & 0.9 & 5.2 & 5.2 & 1.8 \\
\hline Area $\left(\mathrm{mm}^{2}\right)$ & - & $2 \times 1$ & $0.975 \times 0.675$ & $1.5 \times 0.82$ \\
\hline Power $(\mathrm{mW})$ & 390 & 550 & 390 & 200 \\
\hline
\end{tabular}

Conclusions: A 5-bit, 10 GSamples/s T/H circuit is presented. With the input feedthrough cancellation, both the linearity and operation frequency are improved. The proposed $\mathrm{T} / \mathrm{H}$ circuit achieves 5-bit ENOB when analogue input signal frequency is from $250 \mathrm{MHz}$ to $2.5 \mathrm{GHz}$ at $10 \mathrm{GSamples} / \mathrm{s}$ and measured THD is smaller than $-34 \mathrm{~dB}$. It is suitable for high-speed ADCs.

Acknowledgments: The authors thank the Chip Implementation Center (CIC) and the National Science Council (NSC) for chip implementation and support of this work.

C) The Institution of Engineering and Technology 2006 23 January 2006

Electronics Letters online no: 20060247

doi: 10.1049/el:20060247

I.-H. Wang, J.-L. Lin and S.-I. Liu (Graduate Institute of Electronics Engineering and Department of Electrical Engineering, National Taiwan University, Taipei, Taiwan, Republic of China)

E-mail: 1si@cc.ee.ntu.edu.tw

\section{References}

1 Razavi, B.: 'Principle of data conversion system design' (John Wiley \& Sons Inc., 1995)

2 Sato, T., Takagi, S., Fujii, N., Hashimoto, Y., Skata, K., and Okada, H.: ' $4-\mathrm{Gb} / \mathrm{s}$ track and hold circuit using parasitic capacitance canceller'. Eur. Solid-State Circuits Conf. 2004, September 2004, pp. 347-350

3 Nathawad, L.Y., Urata, R., Wooley, B.A., and Miller, D.B.: 'A 40-GHzbandwidth, 4-bit, time-interleaved A/D converter using photoconductive sampling', IEEE J. Solid-State Circuits, 2003, 38, pp. 2021-2030

4 Jiang, X., and Chang, M.F.: 'A 1-GHz signal bandwidth 6-bit CMOS ADC with power-efficient averaging', IEEE J. Solid-State Circuits, 2005, 40, pp. 532-535

5 Jensen, J.C., and Larson, L.E.: 'A broadband 10-GHz track-and-hold in $\mathrm{Si} / \mathrm{SiGe} \mathrm{HBT}$ technology', IEEE J. Solid-State Circuits, 2001, 36, pp. $325-330$

6 Lee, J., Leven, A., Weiner, J.S., Baeyens, Y., Yang, Y., Sung, W.J., Frackoviak, J., Kopf, R.F., and Chen, Y.K.: 'A 6-b 12-GSamples/s trackand-hold amplifier in InP DHBT technology', IEEE J. Solid-State Circuits, 2003, 38, pp. 1533-1538 